\title{
Face-touching behavior as a possible correlate of mask-wearing: A video observational study of public place incidents during the COVID-19 pandemic
}

Lasse S. Liebst ${ }^{1}$, Peter Ejbye-Ernst ${ }^{2,3}$, Marijn de Bruin ${ }^{4,5}$, Josephine Thomas ${ }^{2}$, and Marie R. Lindegaard $^{1,2,3}$

${ }^{1}$ Department of Sociology, University of Copenhagen, Denmark

${ }^{2}$ The Netherlands Institute for the Study of Crime and Law Enforcement, the Netherlands

${ }^{3}$ Department of Sociology, University of Amsterdam, the Netherlands

${ }^{4}$ Radboud University Medical Center, Radboud Institute of Health Sciences, the Netherlands

${ }^{5}$ Corona Behavioural Unit, National Institute for Public Health and the Environment (RIVM), the Netherlands

Corresponding author: Contact: Marie Rosenkrantz Lindegaard, MRLindegaard@nscr.nl

\begin{abstract}
Most countries in the world have recommended or mandated face masks in some or all public places during the COVID-19 pandemic. However, mask-wearing has been thought to increase people's face-touching frequency and, thus, the risk of self-inoculation. Across two studies, we video observed the face-touching behavior of members of the public in Amsterdam and Rotterdam (the Netherlands) during the first wave of the pandemic. Study 1 ( $n=$ 383) yielded evidence in favor of the absence of an association between mask-wearing and face-touching (defined as touches of face or mask), and Study $2(n=421)$ replicated this result. Secondary outcome analysis of the two studies — separately and with pooled datasetsfound a robust negative association between mask-wearing and hand contacts with the face
\end{abstract}


and t-zone (i.e., eyes, nose, and mouth). Our results alleviate the concern that mask-wearing has an adverse face-touching effect.

\section{Introduction}

Mask-wearing in some or all public settings has been widely used as a preventive measure to mitigate the spread of the coronavirus disease 2019 (COVID-19). One concern regarding the use of face masks among members of the public is that mask-wearers may adjust or otherwise touch their mask, thus creating a hand-to-face route for the virus to enter the body through the nose, eyes, and mouth (i.e., the t-zone) (1-3). Fortunately, a small but rapidly growing body of naturalistic observational evidence offers some reassurance, with reports of either no association $(4,5)$ or a negative association $(6,7)$ between mask-wearing and face-touching. For example, in the most extensive study published to date, Chen and colleagues (6) video observed 7586 members of the public across East Asia, Western Europe, and North America, before and during the coronavirus pandemic. They report an overall negative association — especially with respect to t-zone touching —although this association was only significant in the East-Asian countries and not in the Western European or U.S. contexts. This latter null result may, however, reflect a reliance on small subsamples, with very few mask-wearers.

Here, we offer an analysis of the association between mask-wearing and face-touching in an additional Western European context, applying a sample with a more balanced number of persons with and without a mask. To this end, we pre-registered an analysis plan (available at osf.io/bj7tg) and tested the hypothesis in two studies that mask-wearing is negatively associated with face-touching.

\section{Study 1}

\section{Methods}


Data comprised video footage of real-life public behavior in Amsterdam, the Netherlands, captured by public security cameras during the COVID-19 pandemic, between May and the beginning of June 2020 (anonymized data and statistical scripts are available at osf.io/7ek9d). Data were recorded over five days (three Thursdays, one Saturday, and one Sunday) between the morning hours and the early evening. We obtained data from the Amsterdam police with the permission of the Attorney General at the Ministry of Public Affairs. The study was approved by the Ethics Committee for Legal and Criminological Research at Vrije University, and complies with the American Psychological Association's ethics code (Section 8.03) (9), stating that analysis of image data recorded in public places does not require informed consent from those recorded.

The raw sample comprised more than 30,000 hours of raw footage recorded across 50 cameras. From this sample, we selected footage from a single camera, which had a particularly high recording quality and a camera angle that allowed us to follow the passing pedestrians with only negligible breaks in the coverage. We selected only one camera to eliminate potential between-context heterogeneity. Further, we assessed all footage and excluded intervals with technical recording issues (e.g., the recording froze).

Coding procedure. Two trained student research assistants coded data in accordance with a behavioral codebook (ethogram) that we developed with inspiration from ethologists studying animal behavior $(9,10)$. This involved descriptive classifications of recurrent behaviors as observed in the natural, public environment. As part of this, we adapted and assessed the ecological validity of prior behavioral definitions (e.g., face-touching) found in the literature (11). To ensure the epidemiological validity of the face-touching measures, we consulted infectious disease specialists at The National Institute for Public Health and the Environment (the Netherlands). 
The coding began by splitting the eligible footage into 30-minute time segments and then randomly selecting 51 of these. For each selected segment, we planned to sample seven persons with and seven without a face mask (in practice, we could not always satisfy this criterion because of too few mask-wearers per segment). Mask-wearing included individuals wearing medical-grade and homemade masks. We excluded persons wearing face shields, improvised masks (e.g., bandana, scarf), and persons wearing masks covering neither the nose nor the mouth. We also excluded persons who put the mask on or took it off, or changed how it was worn (e.g., from covering both nose and mouth to mouth only). The individuals included in the study are thus either wearing or not wearing a mask for the duration of the observation. This exclusion means that the current data offers insights on 'subconscious' touching (e.g., fidgeting, scratching) rather than 'deliberate' mask repositioning (12). ${ }^{1}$

We observed each person for the duration captured on camera walking through the study setting (although for a maximum of two minutes). The average observation time per individual was 24 seconds $(S D=7.42)$, with a total of 158 person-minutes of observation. In sum, we sampled 176 persons wearing a face mask and 207 not wearing one, comprising a total sample size of 383 persons. This sample size satisfies an a priori statistical power analysis suggesting that 339 observations would detect a small effect $\left(f^{2}=0.05\right)$, with a power of $90 \%$ and a conservative alpha of .005 (13). Note that we coded beyond what the power analysis suggested to have a buffer for incomplete cases (the decision to terminate the sampling procedure was taken before any analyses were conducted). For the inter-rater reliability test of the codebook, we selected 44 individuals and 25 contexts for independent double coding,

\footnotetext{
${ }^{1}$ Note that this exclusion of (in total four) persons who changed mask-wearing status was not part of the initial codebook of Study 1, and, as such, deviates from our pre-registration (see osf.io/bj7tg). However, this was done to align Study 1 with Study 2 in which this exclusion criterion was introduced (because such changes in status proved to be more common here, e.g., due to the implementation of mandatory mask-wearing zones; note that this discovery and decision was made after the coding of the Study 2 videos had begun). To ensure well-defined comparison groups across the studies, we reassessed all persons who touched their mask and excluded those who changed mask-wearing status as part of this mask-touching behavior (this led to the exclusion of an additional six persons in Study 2). It should be stressed that the decision to exclude these cases was implemented prior to examining the potential implications for the results.
} 
with a Krippendorff's alpha $(\alpha)$ larger than .8 as a benchmark for acceptable agreement (scores are reported below) (14).

Measures. The primary face-touching outcome was captured as a binary variable distinguishing between whether or not the person touched his or her facial region-whether covered or uncovered by a mask — at least once with the hand $(\alpha=.89)$ (for illustrations of the face-touching measures, see osf.io/7ek9d). Further, we included three secondary measures with a more narrow definition of face-touching, capturing positive events as direct hand-toface contacts only (i.e., for these outcomes, touching the mask was defined as a non-event). These additional measures captured direct hand contact with the face, the mid-face, or the tzone. The 'face' was defined as including eyes, nose, mouth, ears, cheeks, chin, and forehead $(\alpha=.87)$. The 'mid-face' was restricted to the area from the top of the brows to the tip of the chin with the width of the jaw $(\alpha=1.0)$, and 't-zone' included the eyes, nostrils, and mouth ( $\alpha$ $=.50)$. Note that a low incident rate of the t-zone measure entails an unreasonably low $\alpha$ score despite a high percentage of between-coder agreement (98\%). Gwent's AC1 is considered a more robust interrater statistic for such heavily skewed variables (15), and this test yielded a good score of .98 .

The independent face mask variable captured whether the person wore a face mask covering the nose and mouth, or either the nose or the mouth $(\alpha=1.00)$. We also included a number of controls: visual assessments of the persons' age $(\alpha=.86)$ and sex $(\alpha=.95)$, the number of seconds the person was observed $(\alpha=.94)$, and the level of people-crowding of the segment from where the person was sampled $(\alpha=1.00)$. People-crowding was measured as the number of persons moving through the street per minute. ${ }^{2}$

\footnotetext{
${ }^{2}$ In Study 1, we counted all pedestrians and bicycles passing through an imaginary 'gate' in both directions twice for two minutes per segment (31), and then calculated the average number of people crossing per minute. To make the coding procedure more time efficient, we only counted the number of crossing persons for one minute per segment in Study 2.
} 
Estimation. Data were estimated with linear probability models (using Stata 16's 'reg' module) (16), specified with cluster-robust standard errors to account for the hierarchical data structure (i.e., individuals nested in segments). Given the insight that the traditional alpha level of .05 offers a weak evidential threshold (17), we followed the recommendation to evaluate alpha levels of .05 and .005 as 'suggestive' and 'significant,' respectively (13). All reported $p$-values are two-tailed. Besides $p$-values, we report Bayes factors approximated from Bayesian information criteria (assuming a unit-information prior), which allow for quantification of evidence in favor of the absence of an association (18).

\section{Results}

Across the average observation time of 25 seconds per individual, the incident rate of the dependent variable - measuring touches of the facial region, whether mask covered or not-was $13 \%$ (CI 95\% [0.09, 0.16]). Regarding the secondary outcomes, $10 \%$ (CI 95\% $[0.07,0.13])$ touched their face, $8 \%$ (CI 95\% $[0.05,0.11])$ touched the mid-face, and $5 \%$ (CI $95 \%[0.03,0.08])$ touched the t-zone. These descriptive figures indicate that face-touching is a common occurrence among members of the public (11), while it is also noteworthy that high-risk t-zone touches are the least typical.

Contrary to our pre-specified hypothesis of a negative association, there was no evidence of an association between mask-wearing and touches of the facial region, $\mathrm{B}=-0.01, \mathrm{CI}$ $95 \%[-0.08,0.06], p=.759, \mathrm{BF}_{01}=18.57$, with the Bayes factor suggesting that the $H_{0}$ is approximately 19 times more likely than $H_{\mathrm{a} .}{ }^{3}$ This may be considered positive to strong evidence in favor of the absence of an association, evaluated with the thresholds: weak ( 1-3), positive ( 3-20), strong ( 20-150), very strong (150 or larger) (19).

\footnotetext{
${ }^{3}$ Note that we reached the same nonsignificant result $(\mathrm{B}=-0.007$, CI 95\% [-0.07, 0.08], $\mathrm{p}=.843, \mathrm{BF} 01=19.24)$ if those changing mask-wearing status were re-included in data (see footnote 1).
} 
Next, we examined whether mask-wearing was associated with the three secondary outcomes. Different from the pre-specified analysis, we found suggestive evidence that maskwearing was negatively associated with direct hand contact with the face $(\mathrm{B}=-0.07, \mathrm{CI} 95 \%$ $\left.[-0.12,-0.02], p=.013, \mathrm{BF}_{01}=1.57\right)$, the mid-face $(\mathrm{B}=-0.08, \mathrm{CI} 95 \%[-0.13,-0.03], p=$ $\left..001, \mathrm{BF}_{10}=4.89\right)$, and the t-zone $\left(\mathrm{B}=-0.06, \mathrm{CI} 95 \%[-0.10,-0.01], p=.009, \mathrm{BF}_{01}=1.08\right)$.

The Bayes factors offer evidence for the negative association of mask-wearing with mid-face touching, while data cannot discriminate between $H_{0}$ and $H_{1}$ with respect to the models specified with the outcome variables capturing direct face or t-zone touches. In terms of practical significance, this suggests that mask-wearing is linked with around 6-8 percentage points lower probability for direct hand-to-face contacts. That is a small effect size (equivalent to a 'small' Cohen's $d$, at around between -.20 and -.30 (20)), although the effect may cumulate across time and thus be consequential in the not-very-long run (21). Taken together, these results indicate a negative association between mask-wearing and face-touching, although the robustness of the evidence hinges on how face-touching is operationalized.

\section{Study 2}

\section{Methods}

Study 2 was designed as a replication of Study 1 . There are a few noteworthy between-study differences, however. Specifically, data for Study 2 were collected as part of a larger research project evaluating the implementation of mandatory mask-wearing zones in Amsterdam and Rotterdam. Across these contexts, we collected footage from six locations (rather than from a single camera, located in Amsterdam, as in Study 1). Three of these locations were situated within the zones where the municipalities had made face masks mandatory. The remaining three locations were in areas where the municipalities had considered implementing a mask requirement. The common denominator of the areas was that they were 
above-average busy and crowded pedestrian streets (by comparison, the study location in Study 1 was a more average pedestrian street).

Across the six study contexts, data were selected during 13 days (Wednesdays, Saturdays, and one Sunday) between late July and the end of August (a period when Amsterdam and Rotterdam were facing increasing numbers of recorded COVID-19 cases). From a raw sample of around 480 hours of footage, we randomly selected 78 30-minute segments, across which we conducted 164 person-minutes of observation, with an average observation time of 23 seconds $(S D=17.84)$. Applying the reliability-tested codebook from Study 1, a team of 12 trained student research assistants coded 423 persons (167 with and 256 without a mask).

\section{Results}

Across the average observation time of 23 seconds $(S D=17.84)$ per individual, $20 \%$ (CI 95\% $[0.16,0.24])$ touched their face or mask, $14 \%$ (CI 95\% $[0.11,0.18])$ had direct hand contact with their face, $10 \%$ (CI 95\% [0.07, 0.13]) touched the mid-face, and 5\% (CI 95\% $[0.03,0.07])$ the t-zone. There was a statistically suggestive difference in the proportion of positive cases between Study 1 and Study 2 with respect to touches of the facial region, $z=-$ $2.54, p=.011$. No between-study differences were found with respect to the proportions of direct hand touching of the face $(z=-1.71, p=.088)$, the mid-face $(z=0.84, p=.403)$, or the t-zone $(z=0.15, p=.880)$.

We did not find an association between mask-wearing and touching of the face or mask $\left(\mathrm{B}=0.07, \mathrm{CI} 95 \%[-0.01,0.14], p=0.083, \mathrm{BF}_{01}=4.67\right)$, with the Bayes factor offering positive evidence in favor of the absence of an association. Our secondary outcome analysis found suggestive evidence in favor of negative evidence between mask-wearing and direct touches of the face $\left(\mathrm{B}=-0.07, \mathrm{CI} 95 \%[-0.14,-0.01], p=.032, \mathrm{BF}_{01}=1.79\right)$ and mid-face $(\mathrm{B}$ $\left.=-0.07, \mathrm{CI} 95 \%[-0.14,-0.00], p=.037, \mathrm{BF}_{01}=1.20\right)$, although the corresponding Bayes factors suggest that data cannot discriminate between $H_{0}$ and $H_{1}$. Regarding t-zone touching, we 
found statistically significant evidence of a negative association with mask-wearing $(\mathrm{B}=$ 0.07 , CI 95\% [-0.11, -0.03], $\left.p<.001, \mathrm{BF}_{10}=8.13\right)$, with the Bayes factor offering positive evidence of this link. The effect sizes of mask-wearing on the secondary outcomes are of a small magnitude similar to Study 1. Taken together, both the primary and secondary outcome analyses of Study 2 replicate the overall results of Study 1.

\section{Combined analysis}

\section{Methods}

A prospect of the current datasets is that they may be readily pooled into one large and high-powered dataset (22). A combined ('mega') analysis is a more appropriate approach to synthesize the results across the two studies than to simply 'tally-vote' positive, negative, and null findings (23). Further, given the added statistical power, it is also plausible that this pooled analysis allows for a more accurate estimation of the relatively small-sized and somewhat mixed results across Study 1 and 2, which hinges on model specification choices (24). To explore the most robust patterns across these circumstances, we not only re-ran the few above-specified regression models on the polled data but estimated a large range of regression models across (the 'multiverse' of) other plausible data and model specifications $(25,26)$.

In total, we estimated 4096 alternative specifications (using the 'mrobust' module by 27), including all possible combinations of the following features. First, we included the primary and the three secondary versions of the outcome. Second, the four independent variables, including three additional ones: (a) whether mask-wearing was mandatory or voluntary in the location; (b) whether the person was alone or with someone else (assuming that masks may feel unpleasant during verbal interaction); (c) and the air temperature for each segment (there were large temperature fluctuations across the study periods, with heat waves possibly 
making mask-wearing more of a nuisance). ${ }^{4}$ Third, whether the mask was worn in a manner covering both nose and mouth, or only one of these facial areas. Fourth, we specified models both with and without the persons that we had coded and then decided to exclude because they put their mask on or took it off, or changed how it was worn (see footnote 1). Finally, we ran all combinations with both linear and logistic regression models.

\section{Results}

In Figure 1, we see that across 4096 unique specifications, mask-wearing was negatively associated with face-touching in $75 \%$ of models, when assessed with an alpha level of both .05 and .005 . In contrast, all models $(25 \%)$ that yielded positive estimates were above these alpha thresholds. This contrast between the negative (and significant) and positive (and nonsignificant) estimates across all models was linked to how the outcome was operationalized: While the 512 models specified as touching of face or mask had a positive but nonsignificant estimate, the 512 specifications for each of the secondary outcomes were all negative and below an alpha threshold of both .05 and .005 . These results yield additional and robust evidence in favor of the result found in Study 1 and replicated in Study 2: Mask-wearing has a negative relationship with direct hand-to-face contact, while mask-wearing is not associated with touching of the facial region, whether mask covered or not. ${ }^{5}$

\footnotetext{
${ }^{4}$ The temperature measures were constructed using publicly available data from the Royal Netherlands Meteorological Institute (KNMI). The measurement of whether the person was alone or together with someone had an inter-rater score of .95 .

${ }^{5}$ For full transparency, we note that we found one condition under which the effect of mask-wearing on touches of face or mask was positively associated $(\mathrm{B}=0.09$, CI 95\% $[-0.01,0.17], \mathrm{p}=.024, \mathrm{BF} 01=1.42)$. That is, when Study 2 data were analyzed separately while including the (six) persons who put the mask on or took it off, or changed how it was worn (in line with the pre-registration and see also footnote 1). The p-value for that specification is only suggestive, however, and the relating Bayes factor suggests that $\mathrm{H} 0$ and $\mathrm{H} 1$ cannot be discriminated. Further, this single result does not alter the overall impression of a fragile association. Future research could examine the implications of a full inclusion of this class of persons.
} 
Figure 1.

Sign and significance rates across the multiverse of data and model specifications.

\begin{tabular}{llllll} 
& & \multicolumn{4}{l}{ Versions of the dependent variable } \\
\cline { 3 - 6 } Sign and significance rates & All models & face or mask & face & mid-face & t-zone \\
\hline Positive & $25 \%$ & $100 \%$ & $0 \%$ & $0 \%$ & $0 \%$ \\
Positive and $p<.05$ & $0 \%$ & $0 \%$ & $0 \%$ & $0 \%$ & $0 \%$ \\
Positive and $p<.005$ & $0 \%$ & $0 \%$ & $0 \%$ & $0 \%$ & $0 \%$ \\
Negative & $75 \%$ & $0 \%$ & $100 \%$ & $100 \%$ & $100 \%$ \\
Negative and $p<.05$ & $75 \%$ & $0 \%$ & $100 \%$ & $100 \%$ & $100 \%$ \\
Negative and $p<.005$ & $75 \%$ & $0 \%$ & $100 \%$ & $100 \%$ & $100 \%$ \\
\hline Number of models & 4096 & 1024 & 1024 & 1024 & 1024 \\
\hline
\end{tabular}

\section{Discussion}

The wide use of face masks as a measure against coronavirus disease 2019 raises the question of whether mask-wearing by the general public is linked with adverse face-touching effects. The current paper tested the pre-registered hypothesis that mask-wearing is linked with less face-touching. Contrary to this hypothesis, our analyses of the primary face-touching outcome in Study 1 and 2 both found evidence in favor of a non-association. However, our secondary outcomes analyses suggest that the association with mask-wearing hinges on how face-touching is operationalized - a common but underappreciated experience in statistical research (28). Specifically, across both studies and the combined analysis, we found robust negative estimates of mask-wearing when the outcome was measured as direct hand contact with the face, its mid-zone, or t-zone.

Our results correspond with the prior studies, which either report no association $(4,5)$ or a negative association (7), especially with respect to t-zone touches (6). Taken as a whole, current and prior evidence alleviate the concern that mask-wearing has a positive and adverse face-touching effect (3). The absence of such an effect may be ascribed to how face masks serve as a physical barrier for direct hand-to-face contact or offer a reminder that face touching should be avoided (29). 
One limitation of the current paper is that we are interested in a causal process - do face masks impact face-touching behaviors - while our observational approach merely conveys correlational insights. Accordingly, it may be that unobserved factors, rather than the face mask itself, underpin the negative association with face-touching (e.g., persons who choose to wear a mask may be more careful not to touch their face). Another limitation concerns how generalizable our results—-based on cross-sectional data from public settings in two Dutch cities - are to other countries, social settings, or pandemic phases. Finally, it should be acknowledged that while naturalistic observation of public human behavior has high ecological validity, this method is an often-messy experience involving many ad hoc decisions that may challenge the study's replicability. Just as the current results hinged on how face-touching was defined, it is thus plausible that the mixed findings in the observationallybased literature may be an artifact of different operationalizations. Future research should prioritize the development of reproducible standards via open sharing of codebooks, data, andif it can be done ethically_raw videos (30).

\section{Acknowledgments}

The study was supported by The National Institute for Public Health and the Environment, the Rotterdam and Amsterdam Safety Regions, and the Dutch Organization for Health Research and Innovation (ZonMw grant number: 50-56300-98-603). Further, the authors thank the following persons for their contribution to the coding of the video footage: Juliana Augustinis, Boudewien Bijleveld, Elise Bogaards, Elise Franken, Jim van Hagen, Linda Hak, Manaar el Hijazi, Laura Nollkaemper, Laura Behrend Pedersen, Nina Ranzijn, and Suleyman Tosun.

\section{References}


1. West R, Michie S, Amlot R, Rubin J. Don't touch the T-Zone- - how to block a key pathway to infection with SARS-CoV-2. BMJ Opin. 2020;

2. ECDC. Using face masks in the community - Reducing COVID-19 transmission from potentially asymptomatic or pre-symptomatic people through the use of face masks [Internet]. Stockholm: European Centre for Disease Prevention and Control; 2020 [cited 2020 Aug 13]. Available from: https://www.ecdc.europa.eu/en/publications-data/usingface-masks-community-reducing-covid-19-transmission

3. WHO. Advice on the use of masks in the context of COVID-19: interim guidance, 5 June 2020. World Health Organ [Internet]. 2020 [cited 2020 Aug 17]; Available from: https://apps.who.int/iris/handle/10665/332293

4. Perez-Alba E, Nuzzolo-Shihadeh L, Fonseca-Ruiz A, Aguirre-García GM, HernándezGuedea MA, Perez-Rodriguez E, et al. Frequency of facial touching in patients with suspected COVID-19 during their time in the waiting room. Infect Control Hosp Epidemiol. 2020;1-8.

5. Tao Z, Dong J, Culleton R. The use of facemasks may not lead to an increase in handface contact. Transbound Emerg Dis [Internet]. 2020 [cited 2020 Aug 11];n/a(n/a). Available from: https://onlinelibrary.wiley.com/doi/abs/10.1111/tbed.13698

6. Chen Y-J, Qin G, Chen J, Xu J-L, Feng D-Y, Wu X-Y, et al. Comparison of FaceTouching Behaviors Before and During the Coronavirus Disease 2019 Pandemic. JAMA Netw Open. 2020 Jul 1;3(7):e2016924-e2016924.

7. Lucas TL, Mustain R, Goldsby RE. Frequency of face touching with and without a mask in pediatric hematology/oncology health care professionals. Pediatr Blood Cancer. 2020;67(9):e28593.

8. APA. Ethical principles of psychologists and code of conduct. American Psychological Association; 2010.

9. Eibl-Eibesfeldt I. Human ethology: concepts and implications for the sciences of man. Behav Brain Sci. 1979 Mar;2(1):1-26.

10. Jones LK, Jennings BM, Higgins MK, De Waal FB. Ethological observations of social behavior in the operating room. Proc Natl Acad Sci. 2018;115(29):7575-80.

11. Kwok YLA, Gralton J, McLaws M-L. Face touching: A frequent habit that has implications for hand hygiene. Am J Infect Control. 2015 Feb 1;43(2):112-4.

12. Perl O, Mishor E, Ravia A, Ravreby I, Sobel N. Are humans constantly but subconsciously smelling themselves? Philos Trans R Soc B Biol Sci. 2020 Jun 8;375(1800):20190372.

13. Benjamin DJ, Berger JO, Johannesson M, Nosek BA, Wagenmakers E-J, Berk R, et al. Redefine statistical significance. Nat Hum Behav. 2018 Jan;2(1):6-10.

14. Krippendorff K. Reliability in Content AnalysisSome Common Misconceptions and Recommendations. Hum Commun Res. 2004 Jul 1;30(3):411-33. 
15. Gwet KL. Computing inter-rater reliability and its variance in the presence of high agreement. Br J Math Stat Psychol. 2008;61(1):29-48.

16. Hellevik O. Linear versus logistic regression when the dependent variable is a dichotomy. Qual Quant. 2009;43(1):59-74.

17. Colquhoun D. The reproducibility of research and the misinterpretation of $p$-values. $R$ Soc Open Sci. 2017;4(12):171085.

18. Wagenmakers E-J. A practical solution to the pervasive problems of $\mathrm{p}$ values. Psychon Bull Rev. 2007 Oct 1;14(5):779-804.

19. Raftery AE. Bayesian model selection in social research. Sociol Methodol. 1995;111163.

20. Cohen J. Statistical power analysis for the behavioral sciences. Hillsdale: Erlbaum Associates; 1988.

21. Funder DC, Ozer DJ. Evaluating Effect Size in Psychological Research: Sense and Nonsense. Adv Methods Pract Psychol Sci. 2019 Jun 1;2(2):156-68.

22. Cooper H, Patall EA. The relative benefits of meta-analysis conducted with individual participant data versus aggregated data. Psychol Methods. 2009;14(2):165-76.

23. Hedges LV, Olkin I. Vote-counting methods in research synthesis. Psychol Bull. 1980;88(2):359-369.

24. Gelman A, Carlin J. Beyond power calculations: Assessing type S (sign) and type M (magnitude) errors. Perspect Psychol Sci. 2014;9(6):641-651.

25. Patel CJ, Burford B, Ioannidis JP. Assessment of vibration of effects due to model specification can demonstrate the instability of observational associations. J Clin Epidemiol. 2015;68(9):1046-1058.

26. Steegen S, Tuerlinckx F, Gelman A, Vanpaemel W. Increasing Transparency Through a Multiverse Analysis. Perspect Psychol Sci. 2016 Sep 1;11(5):702-12.

27. Young C. Model Uncertainty and the Crisis in Science. Socius. 2018 Jan 1;4:2378023117737206.

28. Silberzahn R, Uhlmann EL, Martin DP, Anselmi P, Aust F, Awtrey E, et al. Many analysts, one data set: Making transparent how variations in analytic choices affect results. Adv Methods Pract Psychol Sci. 2018;1(3):337-356.

29. Latour B. Pandora's hope: essays on the reality of science studies. Harvard university press; 1999.

30. Gilmore RO, Adolph KE. Video can make behavioural science more reproducible. Nat Hum Behav. 2017;1(7). 
31. Griffiths S. Space syntax as interdisciplinary urban design pedagogy. In: Carmona M, editor. Explorations in urban design: An urban design research primer. Farnham, UK: Ashgate Publishing; 2014. p. 158-67. 